Proceedings of the II Symposium on Applied Nuclear Physics and Innovative Technologies, Kraków, September 24-27, 2014

\title{
Direct Test of T and CPT Symmetries with Entangled Neutral Mesons
}

\author{
A. Di DOMEnico ${ }^{a, b, *}$ \\ ${ }^{a}$ Department of Physics, Sapienza University of Rome, Italy \\ ${ }^{b}$ INFN Sezione di Roma, Rome, Italy
}

\begin{abstract}
In this paper a method to perform direct $\mathrm{T}$ and CPT symmetry tests is briefly discussed, exploiting the Einstein-Podolsky-Rosen correlations of neutral kaon pairs produced at a $\phi$-factory. The KLOE-2 experiment at $D A \Phi N E$, the Frascati $\phi$-factory, could make statistically significant tests of this type.
\end{abstract}

DOI: 10.12693 /APhysPolA.127.1563

PACS: 13.25.Es, 11.30.Er

\section{Introduction}

In general any theoretical connection among the parameters describing $\mathrm{T}, \mathrm{CP}$ or $\mathrm{CPT}$ violation, as given by the CPT theorem, does not imply a corresponding connection among the experimental results of the $\mathrm{T}, \mathrm{CP}$ or CPT tests.

In this paper the possibility to perform a direct test of time-reversal $\mathrm{T}$ symmetry in the neutral kaon system is discussed. It requires the exchange of $i n$ and out states of a transition process. The only existing result [1] for neutral kaons showing a non-zero asymmetry comparing the rates of a reference process $\left(\mathrm{K}^{0} \rightarrow \overline{\mathrm{K}}^{0}\right)$ and its $\mathrm{T}$ conjugated one $\left(\overline{\mathrm{K}}^{0} \rightarrow \mathrm{K}^{0}\right)$ cannot be considered a direct test, because the $\mathrm{T}$ and $\mathrm{CP}$ conjugated processes are identical in this case, and cannot be distinguished ${ }^{\dagger}$. In fact here direct test means a test whose outcome is independent of the result of any other discrete symmetry test, as discussed in detail in Refs. [3-7].

In order to implement such a direct test of the $\mathrm{T}$ symmetry, and also of the CPT symmetry which requires as well the inversion of in and out states, it has been suggested to exploit the Einstein-Podolsky-Rosen (EPR) entanglement of neutral mesons produced at a $\phi$-factory (or B-factory) [4-7]. In fact in this case the initial state of the neutral kaon pair produced in $\phi \rightarrow \mathrm{K}^{0} \overline{\mathrm{K}}^{0}$ decay can be rewritten in terms of any pair of orthogonal states $\left|\mathrm{K}_{+}\right\rangle$and $\left|\mathrm{K}_{-}\right\rangle$:

$$
\begin{gathered}
|i\rangle=\frac{1}{\sqrt{2}}\left\{\left|\mathrm{~K}^{0}\right\rangle\left|\overline{\mathrm{K}}^{0}\right\rangle-\left|\overline{\mathrm{K}}^{0}\right\rangle\left|\mathrm{K}^{0}\right\rangle\right\}= \\
\frac{1}{\sqrt{2}}\left\{\left|\mathrm{~K}_{+}\right\rangle\left|\mathrm{K}_{-}\right\rangle-\left|\mathrm{K}_{-}\right\rangle\left|\mathrm{K}_{+}\right\rangle\right\} .
\end{gathered}
$$

Here one can consider the states $\left|\mathrm{K}_{+}\right\rangle,\left|\mathrm{K}_{-}\right\rangle$defined as follows: $\left|\mathrm{K}_{+}\right\rangle$is the state filtered by the decay into $\pi \pi$ $\left(\pi^{+} \pi^{+}\right.$or $\left.\pi^{0} \pi^{0}\right)$, a pure $\mathrm{CP}=+1$ state; analogously

*e-mail: antonio.didomenico@roma1.infn.it

†' It is worth mentioning that in the neutral B meson system, such a direct $\mathrm{T}$ test has been recently accomplished [2], exploiting a similar methodology as discussed in the following.
$\left|\mathrm{K}_{-}\right\rangle$is the state filtered by the decay into $3 \pi^{0}$, a pure $\mathrm{CP}=-1$ state. Their orthogonal states correspond to the states which cannot decay into $\pi \pi$ or $3 \pi^{0}$, defined, respectively, as:

$$
\begin{aligned}
\left|\widetilde{\mathrm{K}}_{-}\right\rangle & \propto\left[\left|\mathrm{K}_{\mathrm{L}}\right\rangle-\eta_{\pi \pi}\left|\mathrm{K}_{\mathrm{S}}\right\rangle\right], \\
\left|\widetilde{\mathrm{K}}_{+}\right\rangle & \propto\left[\left|\mathrm{K}_{\mathrm{S}}\right\rangle-\eta_{3 \pi^{0}}\left|\mathrm{~K}_{\mathrm{L}}\right\rangle\right],
\end{aligned}
$$

with $\eta_{\pi \pi}=\left\langle\pi \pi|T| \mathrm{K}_{\mathrm{L}}\right\rangle /\left\langle\pi \pi|T| \mathrm{K}_{\mathrm{S}}\right\rangle$ and $\eta_{3 \pi^{0}}=$ $\left\langle 3 \pi^{0}|T| \mathrm{K}_{\mathrm{S}}\right\rangle /\left\langle 3 \pi^{0}|T| \mathrm{K}_{\mathrm{L}}\right\rangle$. With these definitions of states, it can be shown that the condition of orthogonality $\left\langle\mathrm{K}_{-} \mid \mathrm{K}_{+}\right\rangle=0$ (i.e. $\left|\mathrm{K}_{+}\right\rangle \equiv\left|\widetilde{\mathrm{K}}_{+}\right\rangle$and $\left|\mathrm{K}_{-}\right\rangle \equiv\left|\widetilde{\mathrm{K}}_{-}\right\rangle$) corresponds to assume negligible direct $\mathrm{CP}$ (or $\mathrm{CPT}$ ) violation contributions (i.e. $\epsilon^{\prime}, \epsilon_{000}^{\prime} \ll \epsilon$ ), assumption quite well satisfied for neutral kaons (see detailed discussion in Appendix A of Ref. [7]). The validity of the $\Delta S=\Delta Q$ rule is also assumed, so that the two flavor orthogonal eigenstates $\left|\mathrm{K}^{0}\right\rangle$ and $\left|\overline{\mathrm{K}}^{0}\right\rangle$ are identified by the charge of the lepton in semileptonic decays, i.e. a $\left|\mathrm{K}^{0}\right\rangle$ can decay into $\pi^{-} \ell^{+} \nu$ and not into $\pi^{+} \ell^{-} \bar{\nu}$, and vice versa for a $\left|\overline{\mathrm{K}}^{0}\right\rangle$.

Thus, exploiting the perfect anticorrelation of the states implied by Eq. (1), it is possible to have a "flavortag" or a "CP-tag", i.e. to infer the flavor $\left(\mathrm{K}^{0}\right.$ or $\left.\overline{\mathrm{K}}^{0}\right)$ or the $\mathrm{CP}\left(\mathrm{K}_{+}\right.$or $\left.\mathrm{K}_{-}\right)$state of the still alive kaon by observing a specific flavor decay $\left(\pi^{+} \ell^{-} \nu\right.$ or $\left.\pi^{-} \ell^{+} \bar{\nu}\right)$ or CP decay $\left(\pi \pi\right.$ or $\left.\pi^{0} \pi^{0} \pi^{0}\right)$ of the other (and first decaying) kaon in the pair.

In this way one can experimentally access the transitions listed in Table, which can be divided into four categories of events, corresponding to independent $\mathrm{T}, \mathrm{CP}$ and CPT tests.

TABLE

Scheme of possible reference transitions and their associated $\mathrm{T}, \mathrm{CP}$ or $\mathrm{CPT}$ conjugated processes accessible at a $\phi$-factory.

\begin{tabular}{l|c|c|c}
\hline \hline Reference & T-conjug. & CP-conjug. & CPT-conjug. \\
\hline $\mathrm{K}^{0} \rightarrow \mathrm{K}_{+}$ & $\mathrm{K}_{+} \rightarrow \mathrm{K}^{0}$ & $\overline{\mathrm{K}}^{0} \rightarrow \mathrm{K}_{+}$ & $\mathrm{K}_{+} \rightarrow \overline{\mathrm{K}}^{0}$ \\
$\mathrm{~K}^{0} \rightarrow \mathrm{K}_{-}$ & $\mathrm{K}_{-} \rightarrow \mathrm{K}^{0}$ & $\overline{\mathrm{K}}^{0} \rightarrow \mathrm{K}_{-}$ & $\mathrm{K}_{-} \rightarrow \overline{\mathrm{K}}^{0}$ \\
$\overline{\mathrm{K}}^{0} \rightarrow \mathrm{K}_{+}$ & $\mathrm{K}_{+} \rightarrow \overline{\mathrm{K}}^{0}$ & $\mathrm{~K}^{0} \rightarrow \mathrm{K}_{+}$ & $\mathrm{K}_{+} \rightarrow \mathrm{K}^{0}$ \\
$\overline{\mathrm{K}}^{0} \rightarrow \mathrm{K}_{-}$ & $\mathrm{K}_{-} \rightarrow \overline{\mathrm{K}}^{0}$ & $\mathrm{~K}^{0} \rightarrow \mathrm{K}_{-}$ & $\mathrm{K}_{-} \rightarrow \mathrm{K}^{0}$
\end{tabular}




\section{T and CPT symmetry test}

For the direct $\mathrm{T}$ symmetry test one can define the following ratios of probabilities:

$$
\begin{aligned}
& R_{1}(\Delta t)=P\left[\mathrm{~K}^{0}(0) \rightarrow \mathrm{K}_{+}(\Delta t)\right] / P\left[\mathrm{~K}_{+}(0) \rightarrow \mathrm{K}^{0}(\Delta t)\right], \\
& R_{2}(\Delta t)=P\left[\mathrm{~K}^{0}(0) \rightarrow \mathrm{K}_{-}(\Delta t)\right] / P\left[\mathrm{~K}_{-}(0) \rightarrow \mathrm{K}^{0}(\Delta t)\right], \\
& R_{3}(\Delta t)=P\left[\overline{\mathrm{K}}^{0}(0) \rightarrow \mathrm{K}_{+}(\Delta t)\right] / P\left[\mathrm{~K}_{+}(0) \rightarrow \overline{\mathrm{K}}^{0}(\Delta t)\right], \\
& R_{4}(\Delta t)=P\left[\overline{\mathrm{K}}^{0}(0) \rightarrow \mathrm{K}_{-}(\Delta t)\right] / P\left[\mathrm{~K}_{-}(0) \rightarrow \overline{\mathrm{K}}^{0}(\Delta t)\right] .
\end{aligned}
$$

The measurement of any deviation from the prediction $R_{i}(\Delta t)=1$ imposed by $\mathrm{T}$ invariance is a signal of $\mathrm{T}$ violation.

At a $\phi$-factory the corresponding observable quantities are two ratios, $R_{2}^{\exp }(\Delta t)$ and $R_{4}^{\exp }(\Delta t)$ of double decay rates $I\left(f_{1}, f_{2} ; \Delta t\right)$ into decay products $f_{1}$ and $f_{2}$ as a function of the difference of kaon decay times $\Delta t[7,8]$, with $f_{1}$ occurring before $f_{2}$ decay for $\Delta t>0$, and vice versa for $\Delta t<0$. For $\Delta t>0$ one has

$$
\begin{aligned}
& R_{2}^{\exp }(\Delta t) \equiv \frac{I\left(\ell^{-}, 3 \pi^{0} ; \Delta t\right)}{I\left(\pi \pi, \ell^{+} ; \Delta t\right)}=R_{2}(\Delta t) \times D, \\
& R_{4}^{\exp }(\Delta t) \equiv \frac{I\left(\ell^{+}, 3 \pi^{0} ; \Delta t\right)}{I\left(\pi \pi, \ell^{-} ; \Delta t\right)}=R_{4}(\Delta t) \times D,
\end{aligned}
$$

while for $\Delta t<0$ :

$$
\begin{aligned}
& R_{2}^{\exp }(\Delta t)=\frac{D}{R_{3}(|\Delta t|)}, \\
& R_{4}^{\exp }(\Delta t)=\frac{D}{R_{1}(|\Delta t|)} .
\end{aligned}
$$

Here the normalization constant $D$, assuming no CPT violation in semileptonic decays, is $D=$ $\left\{\mathrm{BR}\left(\mathrm{K}_{\mathrm{L}} \rightarrow 3 \pi^{0}\right) \Gamma_{\mathrm{L}}\right\} /\left\{\mathrm{BR}\left(\mathrm{K}_{\mathrm{S}} \rightarrow \pi \pi\right) \Gamma_{\mathrm{S}}\right\}$.

For the direct CPT symmetry test one can define the following ratios of probabilities, similarly as for the T test:

$$
\begin{aligned}
R_{1, \mathrm{CPT}}(\Delta t) & = \\
P\left[\mathrm{~K}^{0}(0)\right. & \left.\rightarrow \mathrm{K}_{+}(\Delta t)\right] / P\left[\mathrm{~K}_{+}(0) \rightarrow \overline{\mathrm{K}}^{0}(\Delta t)\right], \\
R_{2, \mathrm{CPT}}(\Delta t) & = \\
P\left[\mathrm{~K}^{0}(0)\right. & \left.\rightarrow \mathrm{K}_{-}(\Delta t)\right] / P\left[\mathrm{~K}_{-}(0) \rightarrow \overline{\mathrm{K}}^{0}(\Delta t)\right], \\
R_{3, \mathrm{CPT}}(\Delta t) & = \\
P\left[\overline{\mathrm{K}}^{0}(0)\right. & \left.\rightarrow \mathrm{K}_{+}(\Delta t)\right] / P\left[\mathrm{~K}_{+}(0) \rightarrow \mathrm{K}^{0}(\Delta t)\right], \\
R_{4, \mathrm{CPT}}(\Delta t) & = \\
P\left[\overline{\mathrm{K}}^{0}(0)\right. & \left.\rightarrow \mathrm{K}_{-}(\Delta t)\right] / P\left[\mathrm{~K}_{-}(0) \rightarrow \mathrm{K}^{0}(\Delta t)\right] .
\end{aligned}
$$

The measurement of any deviation from the prediction $R_{i, \mathrm{CPT}}(\Delta t)=1$ imposed by CPT invariance is a signal of CPT violation.

At a $\phi$-factory the corresponding observable quantities are, for $\Delta t>0$ :

$R_{2, \mathrm{CPT}}^{\exp }(\Delta t) \equiv \frac{I\left(\ell^{-}, 3 \pi^{0} ; \Delta t\right)}{I\left(\pi \pi, \ell^{-} ; \Delta t\right)}=R_{2, \mathrm{CPT}}(\Delta t) \times D_{\mathrm{CPT}}$,
$R_{4, \mathrm{CPT}}^{\exp }(\Delta t) \equiv \frac{I\left(\ell^{+}, 3 \pi^{0} ; \Delta t\right)}{I\left(\pi \pi, \ell^{+} ; \Delta t\right)}=R_{4, \mathrm{CPT}}(\Delta t) \times D_{\mathrm{CPT}}$,

while for $\Delta t<0$

$$
\begin{aligned}
& R_{2, \mathrm{CPT}}^{\exp }(\Delta t)=\frac{D_{\mathrm{CPT}}}{R_{1, \mathrm{CPT}}(|\Delta t|)}, \\
& R_{4, \mathrm{CPT}}^{\exp }(\Delta t)=\frac{D_{\mathrm{CPT}}}{R_{3, \mathrm{CPT}}(|\Delta t|)} .
\end{aligned}
$$

Here the normalization constant $D_{\mathrm{CPT}}$ is $D_{\mathrm{CPT}}=$ $\left\{\mathrm{BR}\left(\mathrm{K}_{\mathrm{L}} \rightarrow 3 \pi^{0}\right) \Gamma_{\mathrm{L}}\right\} /\left\{\mathrm{BR}\left(\mathrm{K}_{\mathrm{S}} \rightarrow \pi \pi\right) \Gamma_{\mathrm{S}}\right\}$ without any assumption on $\mathrm{CPT}$ violation in semileptonic decays.

The KLOE-2 experiment at DA $\Phi N E$ with an integrated luminosity of $\mathcal{O}\left(10 \mathrm{fb}^{-1}\right)$ [9] could make statistically significant $\mathrm{T}$ and CPT tests, measuring the ratios $R_{2}^{\exp }(\Delta t), R_{4}^{\exp }(\Delta t), R_{2, \mathrm{CPT}}^{\exp }(\Delta t)$ and $R_{4, \mathrm{CPT}}^{\exp }(\Delta t)$ integrated in the statistically most populated $\Delta t$ region, $0 \leq \Delta t \leq 300 \tau_{S}[7]$. In this region these ratios are expected to be constant and a precise knowledge of the normalization constants $D$ and $D_{\mathrm{CPT}}$ is needed in order to not weaken the significance of the test. A precise reconstruction of $\mathrm{K}_{\mathrm{S}} \rightarrow \pi \ell \nu$ and $\mathrm{K}_{\mathrm{L}} \rightarrow 3 \pi^{0}$ decays is needed, and specific studies are in progress at KLOE-2 [10, 11].

\section{Acknowledgments}

I am indebted to J. Bernabeu for several illuminating discussions on the subject. I would like to thank Pawel Moskal and the organizing committee for the invitation to the symposium and the very pleasant stay in Kraków, especially in the Collegium Barnium.

\section{References}

[1] A. Angelopoulos, et al. (CPLEAR collaboration), Phys. Lett. B 444, 43 (1999); Eur. Phys. C 22, 55 (2001).

[2] J.P. Lees, et al. (BaBar Collaboration), Phys. Rev. Lett. 109211801 (2012).

[3] L. Wolfenstein, Int. J. Mod. Phys. E 8, 501 (1999).

[4] M.C. Banuls, J. Bernabeu, Phys. Lett. B 464, 117 (1999); Nucl. Phys. B 590, 19 (2000).

[5] J. Bernabeu, J. Phys. Conf. Ser. 335, 012011 (2011).

[6] J. Bernabeu, F. Martínez-Vidal, P. Villanueva-Pérez, J. High En. Phys. 8, 064 (2012).

[7] J. Bernabeu, A. Di Domenico, P. Villanueva-Perez, Nucl. Phys. B 868, 102 (2013).

[8] Handbook on Neutral Kaon Interferometry at a $\phi$ Factory, Ed. A. Di Domenico, Frascati Phys. Ser., Vol. 43, 2007.

[9] G. Amelino-Camelia et al., Eur. Phys. J. C 68, 619 (2010).

[10] D. Kaminska, Acta Phys. Pol. B 46, 19 (2015).

[11] A. Gajos, Acta Phys. Pol. B 46, 13 (2015). 